\title{
Endoscopic Ultrasound-Guided Celiac Plexus Neurolysis
}

National Cancer Institute

\section{Source}

National Cancer Institute. Endoscopic Ultrasound-Guided Celiac Plexus Neurolysis. NCI

Thesaurus. Code C116514.

The use of endoscopic ultrasound to guide the injection of local anesthetic and alcohol to the celiac plexus, which causes temporary degeneration to the nerve fibers. The procedure is performed in an effort to alleviate intractable plain, generally caused by cancer or chronic pancreatitis. 